•综述・

\title{
基于类胡夢卜素着色的鸟类羽色多样性形成机制
}

薛泊宁, 张雁云, 董路(*

北京师范大学生命科学学院生物多样性与生态工程教育部重点实验室, 北京 100875

摘要: 人们对动物体色的研究由来已久。作为一类让生物呈现出多变色彩的重要色素, 类胡萝卜素可以在鸟类的羽毛、鸟潒 和皮肤等体表组织中沉积, 产生红、橙、黄、粉、紫等颜色。类胡萝卜素不能在鸟类体内合成, 需从食物中摄取, 进而在体 内完成吸收、运输、代谢和沉积等一系列过程，才能用于羽毛着色。与类胡萝卜素着色相关的生理及遗传调控机制一直备受 关注, BCO2、SCARB1和CYP2J19等影响类胡萝卜素在鸟类羽毛中着色的关键基因, 推动了对羽色遗传调控机制的深入认识。 本文介绍了鸟类可利用类胡萝卜素的主要类型和基本特征, 综述了类胡萝卜素着色相关的生理过程以及调控基因研究的最 新进展，旨在增加对鸟类羽毛中类胡夢卜素着色过程和相关遗传机制的理解。

关键词: 类胡萝卜素; 羽色; CYP2J19; BCO2; SCARB1; 代谢

薛泊宁, 张雁云, 董路 (2021) 基于类胡萝卜素着色的鸟类羽色多样性形成机制. 生物多样性, 29, 843-854. doi: 10.17520/biods.2020382. Xue BN, Zhang YY, Dong L (2021) The formation and mechanism of plumage color diversity based on carotenoid pigmentation. Biodiversity Science, 29, 843-854. doi: 10.17520/biods.2020382.

\section{The formation and mechanism of plumage color diversity based on carotenoid pigmentation}

\author{
Boning Xue, Yanyun Zhang, Lu Dong ${ }^{(\mathbb{D} *}$
}

Ministry of Education Key Laboratory for Biodiversity Science and Ecological Engineering, College of Life Sciences, Beijing Normal University, Beijing 100875

\begin{abstract}
Background \& Aims: Animal coloration has long been a topic of interest. As one of the most colorful groups in the world, birds exhibit a wide diversity of plumage pigmentation patterns. Carotenoid, an important component contributing to vivid colors in many avian species, can be deposited in avian integument and produce red, orange, yellow, pink and purple coloration, which can serve as an honest signal of individual condition in mediating social and mating interaction. Therefore, carotenoid pigmentation is an ideal phenotypic trait for understanding the diversity of plumage coloration under a variety of evolutionary pressures and constraints. Most significantly, this complex coloration mechanism provides a variety of opportunities for the evolution of plumage coloration driven by natural and sexual selection. In this review, we highlight the current advances in the mechanisms underlying carotenoid-based coloration in four physiological processes involved in carotenoid coloration in birds. We also introduce the interaction of carotenoid pigment and microstructural coloration, and the ecological forces that drive the evolution of carotenoid coloration.
\end{abstract}

Progresses: We summarized the biochemical pathways of dietary carotenoids absorption, transportation, metabolism and deposition in birds, all of which may affect feather coloration as condition-dependent traits. We further reported a biochemical modification pathway of the yellow dietary carotenoids and conversion to red ketocarotenoids in vivo, which gives birds more opportunities to become colorful. Still, the physiological and genetic mechanisms related to carotenoid processing remain uncertain. With the development of high-throughput sequencing technology, recent breakthroughs have revealed some genes that control carotenoid pigmentation, which has shed light on the molecular basis of carotenoid-based coloration. These genes, such as CYP2J19, BCO2, SCARB1, play an important role in the carotenoid coloration in feathers, and lay the foundation for our understanding of the genetic mechanism of carotenoids 
in the process of feather coloration.

Prospects: This review provides a helpful foundation for understanding the biochemical mechanisms underlying bird coloration. In addition, we have put forward questions in the field that require urgent attention: (1) How do different physiological pathways interact to form and maintain the color diversity in birds? (2) What factors regulate the metabolism and deposition of carotenoids during the growth of bird feathers, which makes the occurrence of carotenoid plumage present a specific temporal and spatial pattern? (3) How does the plumage dichromatism caused by the difference in carotenoid coloration occur and maintain?

Key words: carotenoid; plumage color; CYP2J19; BCO2; SCARB1; metabolism

鸟类是世界上色彩最丰富的生物类群之一, 它 们多变的羽色可通过色素沉积、结构色以及两者的 组合形成(Hill \& McGraw, 2006)。这种复杂的着色 机制为羽色在自然选择和性选择驱动的演化过程 提供了多样的机会(Shawkey \& D’Alba, 2017)。类胡 夢卜素作为鸟类中重要且广泛存在的一类色素, 能 够显示出红、橙、黄、粉、紫等颜色, 并且可以通 过与结构色或其他色素的相互作用, 产生更为广泛 的色彩(Hill \& McGraw, 2006; LaFountain et al, 2015)。与内源合成的黑色素不同, 类胡萝卜素不能 在鸟类体内从头合成, 而需要从饮食中摄取, 进而 通过吸收、运输、代谢以及在体表组织中沉积等过 程, 使鸟类的皮肤、羽毛与喙着色(Weaver et al, 2018)。此外, 一些鸟类可在体内对摄取的类胡萝卜 素进行生化加工, 改变类胡萝卜素的光学特性, 从 而使羽毛呈现出更加丰富的羽色(Koch et al, 2016)。

类胡夢卜素是许多细胞完成基本生命活动必 需的多效性分子。除了用于鸟类羽毛的着色, 类胡 夢卜素还可作为抗氧化剂、免疫调节剂和光保护剂 参与到视觉、免疫等多种重要的生理过程中(Ligon et al, 2016)。一般认为, 与类胡夢卜素相关的性状受 到环境因素(例如食物)的影响, 具有条件依赖性 (condition-dependence), 产生和维持这种性状通常 需要较高的生理代价(Kodric-Brown \& Brown, 1984; von Schantz et al, 1999)。当资源有限时, 类胡萝卜 素在体内的分配与利用会受限于个体自身的健康 程度与生理发育阶段。因此, 与类胡萝卜素相关的 性状通常被作为“诚实”信号来反映个体质量, 这是 性选择理论的重要假说之一(Zahavi, 1977; Hamilton \& Zuk, 1982)。

早期关于鸟类羽色遗传调控机制的研究主要 集中于黑色素, 其生化合成通路相对明确, $M C 1 R$ 、 TYR、ASIP 等黑色素基因会对鸟类羽色的变异产生 重要影响(Tobita-Teramoto et al, 2000; Kerje et al,
2003; Nadeau et al, 2008)。相比而言, 由于对脊椎动 物类胡萝卜素着色的生理过程和调控通路缺乏系 统和深入的认识, 对于类胡夢卜素着色遗传机制的 研究长期止步不前。

近十几年来, 得益于高通量测序技术的发展, 通过比较基因组学与演化发育学研究, 一些与类胡 夢卜素代谢和修饰相关的基因在鸟类中被发现。这 些对鸟类羽毛中类胡萝卜素的着色具有重要作用 的基因, 为我们理解类胡夢卜素在羽毛着色过程中 的遗传调控机制奠定了基础(Lopes et al, 2016; Cuthill et al, 2017; Gazda et al, 2020a)。

本文总结了鸟类中类胡萝卜素着色的最新研究 进展, 从类胡夢卜素的生化特性、着色过程、遗传调 控机制等角度, 综述了鸟类中类胡夢卜素的着色机 制与演化过程, 并展望了未来的研究发展方向。

\section{类胡萝卜素的生化特征与基本类型}

\section{1 类胡夢卜素的生化特征}

作为一类广泛存在的天然色素, 类胡夢卜素几 乎完全由细菌、藻类、真菌以及高等植物合成(Olson \& Owens, 1998)。动物组织中存在的类胡夢卜素属于 $\mathrm{C} 40$ 四萜烯分子, 以交替的 $\mathrm{C}=\mathrm{C}$ 双键为特征, 跨越 由8个异戊二烯单元组成的碳骨架(LaFountain et al, 2015; Toews et al, 2017), 这种碳氢骨架既能以非环 化的形式存在, 也可以在单侧末端或双侧末端进行 环化(惠伯棣, 2005; 高广琦等, 2016)。饮食中大多 数类胡萝卜素的末端都具有含 1 个双键的六碳环(紫 罗酮环, ionone ring), 当这个双键在C5-C6位置, 则 此端环为 $\beta$-紫罗酮环; 当双键在C4-C5位置, 则该 端环为 $\varepsilon$-紫罗酮环(图1A) (Hill \& Johnson, 2012)。

类胡萝卜素为脂溶性, 不溶于水, 其化学结构 直接影响它们呈现出的颜色。类胡萝卜素的光吸收 特性主要取决于具有共轭双键的中心链, 这种化学 结构使类胡夢卜素倾向于吸收短波长(蓝色和紫色) 
(A)

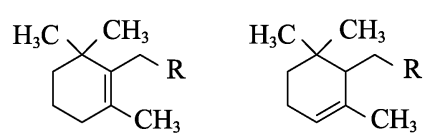

$\beta$-紫罗酮环 $\quad \varepsilon$-紫罗酮环

$\beta$-ionone ring $\varepsilon$-ionone ring

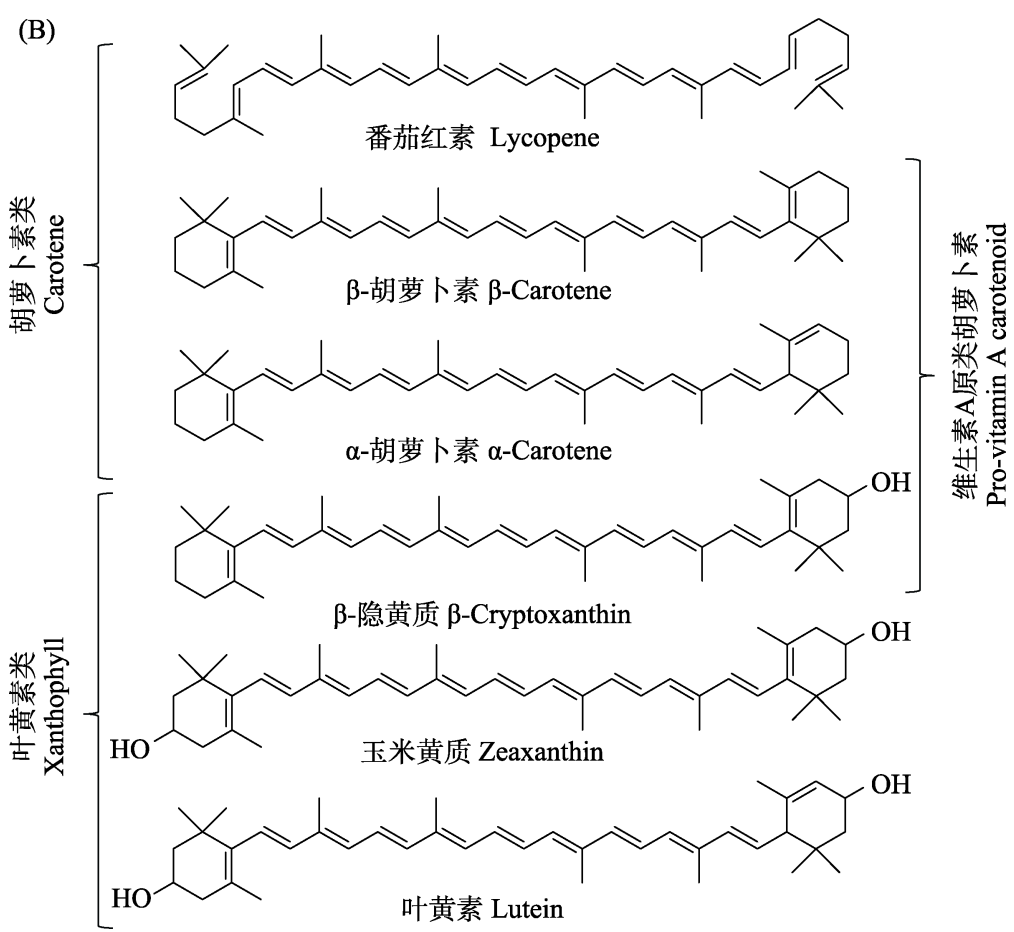

图1 类胡夢卜素的化学结构与分类。(A)类胡夢卜素的两种端环: $\beta$-紫罗酮环与 $\varepsilon$-紫罗酮环; (B)动物中 6 种具代表性的类胡夢 卜素。修改自von Lintig等(2020)。

Fig. 1 Chemical structures and categories of carotenoids. (A) The chemical characteristics of two different end-groups: $\beta$-ionone ring and $\varepsilon$-ionone ring; (B) Six representative carotenoids in animals. Figure adapted from von Lintig et al (2020).

的可见光, 反射出较长波段的光, 使类胡萝卜素呈 现出红色、橙色或黄色(Britton，1995; Olson \& Owens, 1998)。一般认为, 共轭链的长度、双键的数 量以及与其他分子的互作均会影响到类胡萝卜素 的颜色(Britton, 1995; Toews et al, 2017)。类胡萝卜 素吸收光的波长随共轭链增长、双键数量增加而变 短, 发生反射率红移, 故呈现出的颜色更趋近于红 色(Prum et al, 2014)。除此之外, 当类胡萝卜素与相 关蛋白结合时, 会产生紫色、绿色和蓝色等色彩 (Shawkey \& D’Alba, 2017)。鸟类体表组织中的类胡 萝卜素可通过有机溶剂分离出来, 并可使用分光光 度法、光谱法、色谱法等进行分析(Olson \& Owens, 1998)。

\section{2 类胡夢卜素的主要类型}

自然界中存在超过 1,000 种类胡萝卜素, 在动 物组织中已经鉴定出数百种(Britton, 1995; Toews et al, 2017)。鸟类日常摄取的类胡萝卜素虽仅有十几 种, 但一些鸟类可以通过对类胡萝卜素进行内源性 修饰来增加类胡萝卜素的多样性(LaFountain et al, 2015)。到目前为止，鸟类体内已鉴定出30余种类胡 夢卜素分子, 并且仍有新的色素在不同类群中被陆 续发现(Hill \& McGraw, 2006; LaFountain et al, 2010, 2013; Prum et al, 2012)。

已知可用于鸟类着色的类胡夢卜素依据化学 结构可分为两类: 胡萝卜素类(carotene)和叶黄素类 (xanthophyll) (图1B)。第一类是纯碳氢化合物, 主要 包括 $\alpha$-胡萝卜素( $\alpha$-carotene)、 $\beta$-胡萝卜素( $\beta$-carotene) 和番茄红素(lycopene)等; 第二类是氧合类胡萝卜 素, 主要包括叶黄素(lutein)、玉米黄质(zeaxanthin) 和 $\beta$-隐黄质( $\beta$-cryptoxanthin)等(Svensson \& Wong, 2011)。

由于部分类胡夢卜素是脊椎动物合成维生素A 
的主要来源(von Lintig, 2010), 也常据此分为维生 素A原类胡夢卜素(pro-vitamin A carotenoid)和非维 生素A原类胡萝卜素(non pro-vitamin A carotenoid) (Weaver et al, 2018)。前者主要包括 $\alpha$-胡夢卜素、 $\beta$ 胡萝卜素和 $\beta$-隐黄质等, 其碳链末端至少具有一个 未被修饰的 $\beta$-紫罗酮环，因此可裂解合成维生素A; 后者主要包括叶黄素和玉米黄质, 其碳链两端的环 均被氧化, 不具有合成维生素 $\mathrm{A}$ 的活性, 因此不能 作为维生素A的前体(von Lintig et al, 2020)。

\section{2 鸟类利用类胡萝卜素着色的过程}

在鸟类中, 胡夢卜素的着色主要涉及 4 个生理 过程, 包括类胡萝卜素在消化系统中被摄取、在循 环系统中运输、在上皮组织或肝脏中进行代谢, 以 及在体表着色组织中沉积(图2), 其中任一过程出现 遗传变异均有可能会影响到色素最终的沉积, 一些 重要基因的序列突变或表达水平的改变即会对表 型产生巨大的影响(Hill et al, 1994; Hubbard et al, 2010; Orteu \& Jiggins, 2020)。Walsh等(2012)总结了 脊椎动物中与类胡夢卜素着色过程相关的 11 个候 选基因; Gao等(2018)确定了 48个与脂质相关基因 (lipid-related gene)具有直接或间接联系的候选基因, 这些基因可能与鸟羽中广泛的类胡夢卜素沉积有
关。对于一些拥有类胡萝卜素加工和修饰能力的鸟 类，由于长期以来人们对修饰膳食类胡夢卜素的酶 知之甚少, 导致研究这些功能的遗传机制成为一项 艰巨的任务。直到近期，关于类胡萝卜素如何被代 谢为新形式方面才取得了一些新的进展。

类胡夢卜素代谢通路在不同动物类群间具有 生理和遗传上的保守性，由于涉及该过程的基因可 能具有多效性，即部分受体蛋白可能同时会承担其 他基础的生理功能，因此一些鸟类中类胡夢卜素相 关的表型多态性也有可能是由基因的调节突变 (regulatory mutation)引起的，不涉及到基因功能的 完全丧失(Van der Horst et al, 2009; Walsh et al, 2012)。

\section{1 类胡夢卜素的吸收与运输}

类胡夢卜素的吸收发生在胃肠道中，是指类胡 萝卜素向淋巴或血液运动的过程, 其中类胡夢卜素 从食物基质中释放以及在脂质相中溶解是吸收过 程中的关键步骤(Erdman et al，1993; Yeum \& Russell，2002)。类胡夢卜素是高度疏水的化合物， 它们不能直接溶解于缺乏两亲性分子的水性介质, 因此，饮食中的脂质可以很好地帮助类胡萝卜素分 散到胃肠道中，继而以乳状液(emulsion)的形式散 布在消化液中(Kotake-Nara \& Nagao, 2011)。在胆计

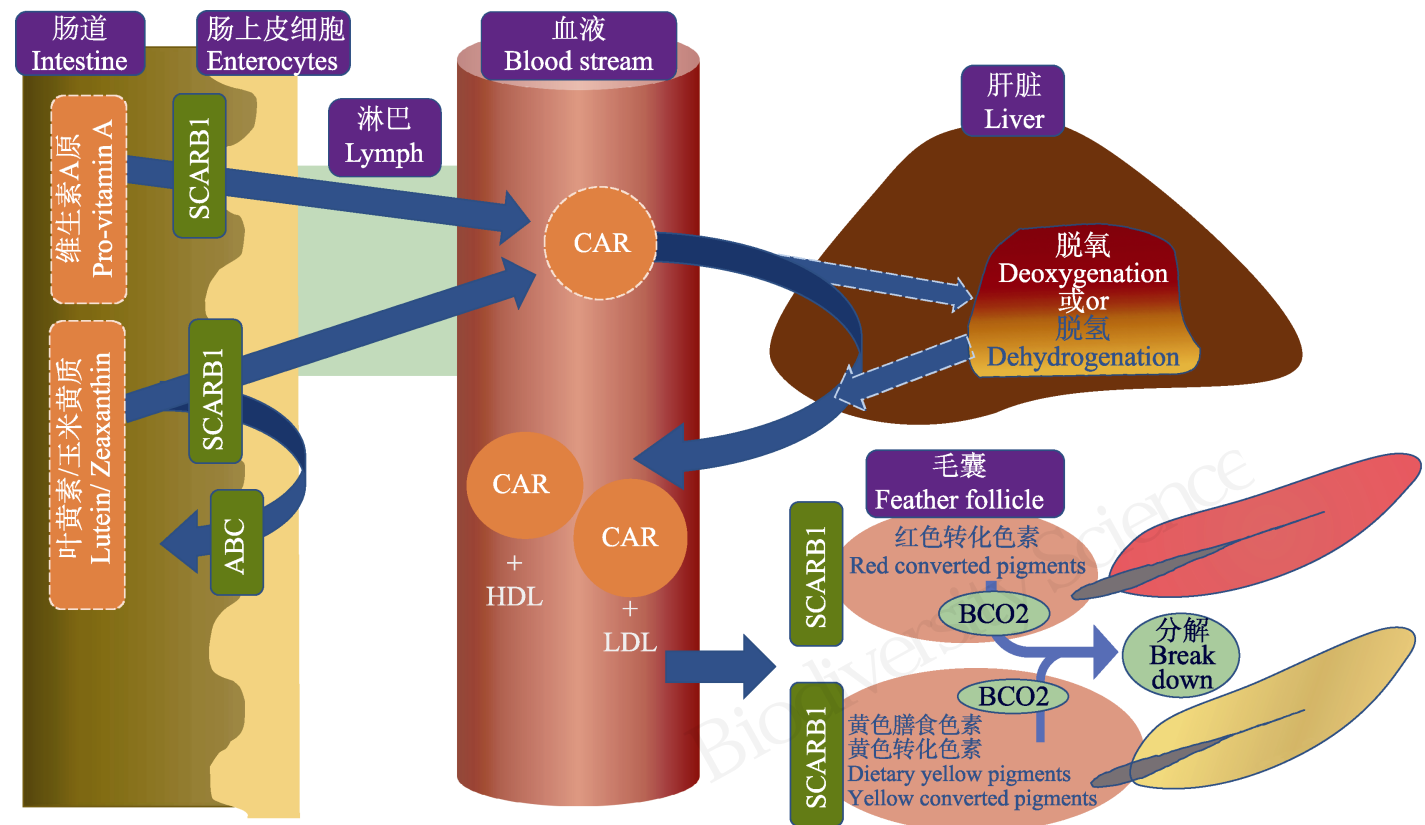

图2 类胡夢卜素在羽毛中着色的主要过程。修改自Yeum和Russell (2002), Hill和Johnson (2012)。

Fig. 2 Main process of carotenoid-based coloration in bird feathers. Figure adapted from Yeum \& Russell (2002) and Hill \& Johnson (2012). 
和脂肪分解酶的作用下, 类胡夢卜素被溶解在由游 离脂肪酸、胆汁盐、磷脂和单酰基甘油构成的混合 胶束(mixed micelle)中(Nagao, 2011)。随后, 胶束形 式的类胡萝卜素在肠上皮跨膜转运蛋白作用下, 吸 收进入肠上皮细胞(Scita et al, 1993)。脊椎动物与类 胡萝卜素结合的转运蛋白主要包括清道夫受体蛋 白家族的SCARB1 (SR-B1)与CD36以及ABC转运蛋 白家族的 ABCG5/G8 复合体等 (Yu et al, 2004; During et al, 2005; Kotake-Nara \& Nagao, 2011)。清 道夫受体蛋白家族是具有胞外结构域的糖基化跨 膜蛋白, 包含大型的疏水通道, 其中SCARB1的转 运功能在果蝇、金丝雀(Serinus canaria)等物种中均 得到了证实(Kiefer et al, 2002; Toomey et al, 2017)。 ABCG5/G8复合体仅在肠上皮细胞和肝细胞中表达, 在限制膳食固醇吸收的过程中起到重要作用, 并且 该功能在哺乳动物中已得到验证( $\mathrm{Yu}$ et al, 2004); ABCG5在人类中的多态性与叶黄素的生物利用度 有关, 并且ABCG5可能参与到叶黄素和玉米黄质 反向运输至肠腔的过程中, 该过程通过耗能的主动 运输来完成(Herron et al, 2006)。

在鸟类的肠上皮黏膜细胞中, 类胡萝卜素被脂 蛋白包装成乳糜微粒(chylomicron)分泌到淋巴, 继 而进入血液循环系统 (During et al, 2005; Kotake-Nara \& Nagao, 2011)。在血液中, 乳糜微粒 被脂蛋白脂肪酶降解, 类胡萝卜素残余到达肝脏进 行咜存、代谢及包装再分泌(Yeum \& Russell, 2002)。 肝脏再分泌的类胡萝卜素需要与脂蛋白(lipoprotein) 结合进入血液循环中(Parker, 1996), 其中胡夢卜素 类在血浆中的运输需要与低密度脂蛋白 (low density lipoprotein, LDL)结合, 其他极性较强的类 胡夢卜素(例如叶黄素类)则倾向与高密度脂蛋白 (high density lipoprotein, HDL)结合(Erdman et al, 1993)。鸟类肝脏中的转运蛋白ABCA1负责向肝外 输出类胡夢卜素和其他脂类, 该过程对于类胡萝卜 素与HDL结合是重要的限速步骤, 可能影响到类胡 夢卜素着色。通过对具有白色皮肤的家鸡“WHAM” 突变体的研究, 发现其 $A B C A 1$ 基因出现错义突变, 虽然饮食中的叶黄素和玉米黄质仍可被肠道和肝 正常吸收, 但从肝脏向外周组织转运的过程显著缺 失, HDL水平显著降低, 最终导致白色皮肤表型产 生(Attie et al, 2002; Connor et al, 2007)。

脂蛋白被靶组织摄取是一个有选择的过程, 通
常需要被靶组织(皮肤、毛囊、视网膜等)上的受体 (例如SCARB1与CD36)识别, 继而以被动运输的方 式被靶组织摄取, 促进类胡萝卜素向细胞内的转运 (Sakudoh et al, 2013)。SCARB1可作为高密度脂蛋白 受体来介导靶组织类胡萝卜素着色, 其编码基因 SCARB1发生突变可能会破坏类胡萝卜素的转运过 程。Toomey等(2017)发现金丝雀的白色隐性突变个 体与红色或黄色的品种相比, 其组织中类胡夢卜素 的含量非常低。结合遗传图谱法, 发现白色隐性等 位基因是由于 SCARB1的剪切供体存在点突变, 导 致数量最多的剪切异构体出现异常剪切, 丧失了转 运类胡萝卜素的功能, 最终导致类胡夢卜素无法在 体表组织中积累着色。

\section{2 类胡夢卜素的代谢与沉积}

体表组织选择性摄取的类胡萝卜素以脂滴的 形式积累, 在羽毛中与角蛋白结合, 呈现出黄或红 等颜色(Brush, 1990)。这些体表衍生物既可以直接 从食物中获取、沉积未经修饰的黄色膳食类胡萝卜 素(dietary carotenoid), 或者沉积内源修饰后的类胡 萝卜素, 获得更丰富的颜色(Stradi et al, 1996)。例如, 绝大部分雀形目的红色羽毛都是黄色膳食类胡萝 卜素内源转化为红色酮基类胡萝卜素 (ketocarotenoid)的结果(Davies, 1985)。McGraw等 (2006) 提出雀形目中类胡萝卜素的着色机制存在3 个层次:

(1)沉积黄色腼食色素(dietary yellow pigments): 将未经修饰的黄色类胡夢卜素(例如叶黄素和玉米 黄质)沉积到皮肤、羽毛和鸟喙中(Hill \& McGraw, 2006)。这是色素沉积中最简单的机制, 部分蜡嘴雀 和森莺等类群会通过这种方式使羽毛呈现黄色(Hill \& Johnson, 2012)。

(2)沉积黄色转化色素 (yellow converted pigments): 将黄色类胡夢卜素在体内转化为其他黄色 色素的途径, 包括将膳食叶黄素和玉米黄质, 经过 脱氢生成3-脱氢叶黄素(3-dehydrolutein)以及金丝雀 黄素(canary xanthophyll) A和B (Hill \& McGraw, 2006)。3-脱氢叶黄素在肝脏中生成, 金丝雀黄素生 成位置尚不清楚, 推测在靶组织(例如毛囊)中合成 (Hill \& Johnson, 2012)。美洲金翅雀(Carduelis tristis) 会采用此种方式来生成并在羽毛中沉积金丝雀黄 素A和B (McGraw et al, 2005; Weaver et al, 2018)。

(3)沉积红色转化色素(red converted pigments): 
酮化酶(ketolase)将黄色类胡萝卜素氧化成红色的 酮基类胡夢卜素。这个过程涉及的代谢机制比较复 杂, 并已在多种红色的雀形目鸟类中被发现(Hill \& McGraw, 2006)。3-差基海胆酮(3-hydroxy-echinenone)是金翅雀亚科最主要的酮基类胡萝卜素，它 是 $\beta$-隐黄质中一个 $\beta$-紫罗酮环被氧化后的产物。雄 性美洲家朱雀(Haemorhous mexicanus)的红色羽毛 主要以3-差基海胆酮进行着色, 其个体间黄色到红 色的变化与酮基类胡萝卜素的含量成正相关 (Inouye et al, 2001; McGraw et al, 2006)。在鸟类羽毛 中发现的其他酮基类胡萝卜素还包括海胆酮 (echinenone)和角黄素(canthaxanthin), 它们分别是 $\beta$-胡萝卜素一个或两个端环被氧化的产物, 人工培 育的红色型金丝雀利用角黄素进行着色(Lopes et al, 2016)。在其他鸣禽中, 由玉米黄质两个端环氧化形 成的虾青素, 也是常见的一种红色酮基类胡夢卜 素。雄性猩红比蓝雀(Piranga olivacea)的红色繁殖 羽为虾青素着色 (Hudon, 1991); 雄性主红雀 (Cardinalis cardinalis)的红色羽毛则由虾青素、角黄 素、 $\alpha$-皮黄素 ( $\alpha$-doradexanthin) 与金戞花红素 (adonirubin)等共同沉积着色(McGraw et al, 2003)。

酮化酶修饰类胡萝卜素的过程一般被认为发 生在肝脏与视网膜中, 也有研究认为该过程也会在 毛囊等沉积部位发生(del Val et al, 2009; Ge et al, 2015; Mundy et al, 2016)。Hill等(2019)通过对美洲家 朱雀的肝线粒体进行生理学测量, 以及对酮化酶进 行分子建模, 证实类胡萝卜素的酮化发生于线粒体 内膜, 且红色类胡萝卜素羽色与线粒体的功能和活 性密切相关。

尽管类胡萝卜素的内源性修饰早在数十年前 已被提出, 但相应的功能基因仍不清楚。近年来两 项研究独立地发现了同一种编码细胞色素P450酶 的基因-CYP2J19, 可以将黄色的膳食类胡萝卜 素转化为红色的酮基类胡萝卜素, 是影响鸟类着色 的重要酮化酶基因(Lopes et al, 2016; Mundy et al, 2016)。Mundy等(2016)以具有黄色喙部的斑胸草雀 (Taeniopygia guttata) 突变体为研究对象, 利用不同 表型个体的基因组SNP数据, 通过连锁图谱(linkage mapping)将决定颜色差异的关键突变定位于 8 号染 色体的CYP2J19基因座。CYP2J19在突变体的喙部 几乎不能表达, 导致突变体具有黄色的喙, 而不是 野生型红色的喙, 证实CYP2J19是类胡萝卜素酮化
酶的重要候选基因; Lopes等(2016)使用金丝雀与黑 头红金翅雀(Spinus cucullata)的杂交后代一一红色 型金丝雀(red-factor canary)鉴定出相同的候选基因, 并发现CYP2J19的表达量在红色型金丝雀的皮肤和 肝脏中显著上调, 进一步证实CYP2J19是介导鸟类 红色类胡夢卜素着色的关键酮化酶基因。此外, 酮 化酶催化产生的酮基类胡夢卜素不仅会使羽毛呈 现红色, 同时也贡献于鸟类红色视网膜油滴, 因此 该基因在视觉系统中功能保守 (Twyman et al, 2018a)。

尽管编码酮化酶的基因已被揭示, 但是有关 CYP2J19参与的着色机制在不同物种中的普遍性未 完全得到验证。首先，该基因在不同物种间的拷贝 数存在差异, 例如在红色型金丝雀中, CYP2J19为 单拷贝基因, 而在斑胸草雀中为双拷贝。其次, 在 不同物种中的高表达部位也存在差异。红色型金丝 雀中的肝脏和皮肤都可以检测到CYP2J19的高表达; 而在斑胸草雀中, CYP2J19主要在红色的喙部表达; Twyman等(2018b)通过对16种红色与黄色非洲织布 鸟的比较研究, 发现CYP2J19在红色织布鸟肝部的 表达量要显著高于其他色型的织布鸟, 但在皮肤中 几乎检测不到该基因的表达, 表明鸟类还可以通过 增加CYP2J19在肝脏中的表达量来提升体内酮基类 胡夢卜素的含量, 进而形成红色的羽毛。此外, 通 过对红背细尾絮茴(Malurus melanocephalus)的研究 发现睪酮可以上调CYP2J19的表达水平, 增强酮基 类胡夢卜素的代谢与着色能力, 从而提升了个体所 获得的性选择优势与繁殖成功率, 为理解内分泌信 号对CYP2J19基因表达及羽色的调控机制提出了新 的证据(Khalil et al, 2020)。

鸟类体内的类胡萝卜素处于参与沉积着色与 分解的动态过程中, $\beta$-胡萝卜素加氧酶( $\beta$-carotene oxygenases) 作为参与类胡萝卜素分解的一类酶, 在 不同动物中被广泛研究, 对类胡萝卜素的代谢具有 重要影响。到目前为止, 在哺乳动物和鸟类中已经 鉴定出两个 $\beta$-胡萝卜素加氧酶基因: $B C O 1$ 和 $B C O 2$, 其中BCO1酶可通过裂解维生素A原类胡萝卜素, 生成维生素A用于视觉系统与免疫系统; 与BCO1 酶相比, BCO2酶除了可以裂解维生素A原类胡萝卜 素, 还可以裂解更多种的类胡夢卜素, 是调节类胡 夢卜素沉积的重要候选基因(Våge\& Boman, 2010)。

$B C O 1$ 和 $B C O 2$ 发生突变对多种生物中的类胡 
萝卜素代谢具有重要影响, 这些突变可能会导致动 物组织中类胡萝卜素的积累增加, 从而产生色素积 累的表型, 直接影响动物的着色 (Toews et al, 2017)。Eriksson等(2008)证明家鸡腿部的黄色皮肤 是由 $B C O 2$ 顺式调控元件的突变所引起。该突变抑 制了 $\mathrm{BCO} 2$ 在家鸡皮肤中的表达, BCO2酶无法把黄 色类胡萝卜素分解为无色产物, 导致类胡萝卜素在 家鸡腿部皮肤中的积累。该研究认为家鸡的黄腿性 状是由同为黄腿的姊妹种—灰原鸡 (Gallus sonneratii) 基因渗入而获得的, 打破了长期以来关 于红原鸡是家鸡唯一祖先的固有认知。不同于上述 $B C O 2$ 的突变形式, 在对具有红色潒部的金丝雀突 变体进行的研究中, $B C O 2$ 的单个非同义突变会消 除BCO2酶的裂解活性, 导致红色类胡夢卜素在喙 部积累(Gazda et al, 2020b)。除此之外, 近缘种间或 两性别间的羽色差异也可能与 $B C O 2$ 有关。通过对 两种在类胡夢卜素着色区域呈现出羽色差异的森 芶进行比较基因组分析, 发现 $B C O 2$ 位于少数的高 分化区域中, 表明该基因很可能与类胡萝卜素着色 差异有关(Toews et al, 2016); 在人工培育的马赛克 型金丝雀(mosaic canary)雌雄个体中, BCO2在体表 组织中活性不同, 导致类胡夢卜素在性别间差异性 降解, 从而产生性二态现象(Gazda et al, 2020a)。

值得注意的是, 尽管与 $B C O 2$ 相关的性状看似 容易获得和丢失, 增添了表型的多样性和不稳定性, 然而, BCO2酶功能缺失或减弱的个体可能需面临 类胡萝卜素在器官中过度聚积的风险。例如在对美 洲金翅雀的研究中发现, 高浓度类胡萝卜素会导致 其体内的肌酸激酶增加, 加速骨骼肌衰竭, 对其飞 行能力造成影响(Huggins et al, 2010)。因此, 在考虑 BCO2突变带来的鲜艳羽色在性选择中的优势时, 同时也需要考虑过度积累类胡萝卜素需承担的生 理代价。

\section{类胡萝卜素色素与羽毛微结构的互作}

类胡夢卜素除了可以单独使鸟类着色外, 在某 些情况下, 还可以和羽毛的微结构发生相互作用, 增加鸟类羽毛着色的方式。Shawkey和D’Alba (2017) 提到类胡萝卜素与结构互作的3种情况, 会对鸟类 呈现出的颜色起到潜在影响, 它们分别为: (1)无序 的角蛋白微结构会对类胡夢卜素着色产生影响; (2) 有序的微结构会对类胡萝卜素着色产生影响; (3)类
胡萝卜素与其他蛋白质的相互作用。

鸟类的羽毛是由 $\beta$-角蛋白构成的纤维性蛋白多 聚物, 类胡萝卜素沉积于角蛋白形成的角质层中, 产生颜色(郑光美, 2012)。由于羽毛的蛋白结构和色 素均会与入射光线发生相互作用, 角蛋白中较厚的 随机气孔阵列会散射更多光线, 从而增加羽毛的亮 度, 因此有些羽毛即使呈现出典型的色素色, 仍可 能兼具结构色成分(Shawkey \& D'Alba, 2017)。例如， 美洲金翅雀亮黄的羽色是类胡萝卜素与通过角蛋 白增强反射的光线共同作用产生的(Shawkey \& Hill, 2005), 这是无序角蛋白结构影响鸟类类胡萝卜素 着色的典型案例。

有序的纳米结构包括规则排布的海绵状角蛋 白或黑素体等。鸟类羽毛非虹彩的绿色主要由该着 色模式产生, 例如绿阔嘴鸟(Calyptomena viridis)呈 现出的绿色由黄色类胡夢卜素与准有序的角蛋白 阵列共同作用产生(Prum et al, 2014); 此外, 部分鸟 类中暗绿的羽色由分布在羽毛不同位置的类胡萝 卜素与黑素体结合的方式产生。黑素体指表皮色素 细胞中分泌形成的黑色素颗粒, 其形态种类多样, 可为实心或空心、球状或棒状、扁平或圆形等, 并 可以形成单层薄膜或多边形阵列等结构, 使得入射 光在黑素体与其他材质的界面发生散射(Maia et al, 2012)。黑素体的着色模式在鹦鹉中最为典型。鹦武 摄取膳食类胡夢卜素, 但并不把它们沉积在羽毛中, 而是沉积一类谱系特有的黄色多烯醛色素——樱 鸫黄素(psittacofulvins) (Cooke et al, 2017; Mundy, 2018)。在不同颜色的虎皮鹦鹉 (Melopsittacus undulatus)中, 除黄色和灰色个体外, 其他所有色型 的羽毛在角质层上覆盖了一层黑素体, 因此鹦鹉黄 素与这种结构发生互作后增加了色彩饱和度, 并可 以产生更为广泛的色彩(D’Alba et al, 2012)。由于 鹦 绒黄素和类胡夢卜素都属于脂色素, 因此以上羽色 形成机制对研究类胡夢卜素与黑色素相互作用对 羽色的影响具有重要启发。

羽毛中的类胡夢卜素通常会结合蛋白质来保 证其化学性质的稳定, 当类胡萝卜素与结构蛋白形 成复合物时, 原本的黄色、橙色或红色可以变为蓝 色、紫色或绿色(Weesie et al, 1995)。例如在呈现出 鲜亮红色的美洲红噮(Eudocimus ruber)、橙红色的 玫红丽唐纳雀(Piranga rubra)与具有紫色羽毛的白 眉紫须伞岛(Iodopleura isabellae)中, 红色、橙红与 
紫色羽毛中的类胡夢卜素均主要为角黄素, 而不同 的蛋白质-角黄素结合模式导致了羽毛反射光谱的 变化(Mendes-Pinto et al, 2012)。

\section{4 类胡萝卜素羽色的演化历史及驱动力}

由于类胡萝卜素的易降解性, 在鸟类羽毛化石 中尚未发现类胡萝卜素着色的证据。对于类胡萝卜 素羽色演化历史的认识主要来源于系统发育研究。 基于全球所有鸟类羽色重建类胡萝卜素着色的演 化历史, 发现类胡萝卜素着色在鸟类中经历了多次 起源, 最早一次起源发生于约 5,600万年前(古新世) 雀形目的共同祖先。然而, 在雁形目、鹳形目等十 余个已经证实存在类胡萝卜素羽色的类群中, 类胡 夢卜素着色的能力是在中新世以后独立演化而来 的(Thomas et al, 2014; Twyman et al, 2016), 这暗示 现生鸟类在羽毛中沉积类胡萝卜素的能力可能是 趋同演化的结果。在现存鸟类中, 雀形目利用类胡 夢卜素着色的物种比例最高, 演化历史最长, 是当 前研究关注的重点类群。基于鸟类羽色来源的成分 鉴定与祖先状态重建分析, 发现雀科的祖先最有可 能先利用未经修饰的膳食类胡萝卜素对羽毛进行 着色, 此后藉由类胡萝卜素关键代谢基因的突变, 部分物种获得了在体内对类胡萝卜素进行修饰的 能力, 它们就不会再将膳食类胡萝卜素直接用于羽 毛着色(Ligon et al, 2016), 这种渐进式的过程表明, 类胡萝卜素羽色的形成与调控机制经历了由简单 到复杂的多个演化阶段。

类胡萝卜素着色一直以来都被认为是性选择 研究的经典模式(Morehouse, 2014; Weaver et al, 2018)。比较系统发育分析表明, 霸暡小目雄性羽色 的快速进化是驱动类胡萝卜素羽色多样化的主要 动力, 表明种间性选择信号的差异与类胡萝卜素羽 色的演化模式具有显著相关性(Cooney et al, 2019)。 昂贵信号理论(costly signaling principle)提出的资源 权衡假说(resource trade-off hypothesis, RTOH)是对 类胡萝卜素羽色作为诚实性选择信号的重要解释 (Weaver et al, 2017)。基于该假说, 用于着色的类胡 萝卜素会占用原本用于对抗细胞活性氧(ROS)的抗 氧化资源, 因此只有高质量雄性才能负担资源分配 的代价一一在产生鲜艳色彩信号的同时, 仍然可以 满足正常生存的需求(Morehouse, 2014)。然和，对于
类胡萝卜素羽色能否作为诚实信号尚未得到一致 认可。有观点认为其他分子也可承担抗氧化剂的功 能, 减少类胡萝卜素的消耗, 弱化了类胡萝卜素羽 色作为 “诚实信号” 的作用 (Hartley \& Kennedy, 2004); 甚至关于类胡萝卜素资源是否“昂贵”的问 题至今也还存在争议(Hill \& Johnson, 2012; Koch et al, 2018)。Hill (2011) 提出了新的共享通路假说 (shared-pathway hypothesis), 如果类胡夢卜素代谢 与细胞呼吸效率之间存在密切的生理联系, 则可以 直接解释许多类胡萝卜素性状的演化, 而不完全受 限于资源权衡假说(Johnson \& Hill, 2013)。由于红色 酮基类胡萝卜素合成的过程与线粒体密切相关, 因 此与其相关的性状尤其适合检验共享通路假说, 近 来对斑胸草雀和红交嘴雀(Loxia curvirostra)的研究 也支持了共享通路假说的预期(Cantarero et al, 2020a, b)。

\section{5 展望}

类胡萝卜素着色的相关表型是鸟类社交的重 要信号, 也是理解鸟类羽饰在多种演化压力与制约 因素下多样化的理想表型。近年来依赖于高通量测 序技术, 在鸟类中新发现的类胡萝卜素基因型-表 型对应关系, 使得类胡萝卜素着色的遗传机制黑箱 被逐步打开, 将为基于类胡萝卜素着色表型的性选 择与自然选择研究提供更充分的科学证据。尽管如 此, 我们对控制类胡萝卜素信号遗传机制的理解仍 不完善, 例如: (1)个体内不同的生理途径如何相互 作用, 以形成并维持这些信号; (2)在鸟类羽毛的生 长过程中, 类胡萝卜素的代谢和沉积过程受到何种 因素的调控, 使类胡夢卜素羽色的发生呈现出特定 的时空规律; (3)由类胡萝卜素着色差异引起的羽色 性二态如何发生并维持等。随着功能基因组学与进 化发育生物学研究方法的发展, 我们相信未来有望 发现更多与类胡萝卜素着色相关的功能基因与代 谢、调控通路, 有助于揭开鸟类利用类胡萝卜素着 色的演化历史与遗传机制的全貌, 从而加深我们对 鸟类羽色多样性起源与维持机制的理解, 更好地认 识自然选择与性选择对鸟类表型演化的作用。

致谢: 衷心感谢两位匿名审稿专家及编委对本文提 出的宝贵意见和建议。 


\section{ORCID}

董路 (D) https://orcid.org/0000-0001-9476-3321

\section{参考文献}

Attie AD, Hamon Y, Brooks-Wilson AR, Gray-Keller MP, MacDonald MLE, Rigot V, Tebon A, Zhang LH, Mulligan JD, Singaraja RR, Bitgood JJ, Cook ME, Kastelein JJP, Chimini G, Hayden MR (2002) Identification and functional analysis of a naturally occurring E89K mutation in the ABCA1 gene of the WHAM chicken. Journal of Lipid Research, 43, 1610-1617.

Britton G (1995) Structure and properties of carotenoids in relation to function. The FASEB Journal, 9, 1551-1558.

Brush AH (1990) Metabolism of carotenoid pigments in birds. The FASEB Journal, 4, 2969-2977.

Cantarero A, Andrade P, Carneiro M, Moreno-Borrallo A, Alonso-Alvarez C (2020a) Testing the carotenoid-based sexual signalling mechanism by altering CYP2J19 gene expression and colour in a bird species. Proceedings of the Royal Society B: Biological Sciences, 287, 20201067.

Cantarero A, Mateo R, Camarero PR, Alonso D, Fernandez-Eslava B, Alonso-Alvarez C (2020b) Testing the shared-pathway hypothesis in the carotenoid-based coloration of red crossbills. Evolution, 74, 2348-2364.

Connor WE, Duell PB, Kean R, Wang YM (2007) The prime role of HDL to transport lutein into the retina: Evidence from HDL-deficient WHAM chicks having a mutant ABCA1 transporter. Investigative Ophthalmology \& Visual Science, 48, 4226-4231.

Cooke TF, Fischer CR, Wu P, Jiang TX, Xie KT, Kuo J, Doctorov E, Zehnder A, Khosla C, Chuong CM, Bustamante CD (2017) Genetic mapping and biochemical basis of yellow feather pigmentation in budgerigars. Cell, 171, 427-439.

Cooney CR, Varley ZK, Nouri LO, Moody CJA, Jardine MD, Thomas GH (2019) Sexual selection predicts the rate and direction of colour divergence in a large avian radiation. Nature Communications, 10, 1773.

Cuthill IC, Allen WL, Arbuckle K, Caspers B, Chaplin G, Hauber ME, Hill GE, Jablonski NG, Jiggins CD, Kelber A, Mappes J, Marshall J, Merrill R, Osorio D, Prum R, Roberts NW, Roulin A, Rowland HM, Sherratt TN, Skelhorn J, Speed MP, Stevens M, Stoddard MC, Stuart-Fox D, Talas L, Tibbetts E, Caro T (2017) The biology of color. Science, 357, eaan0221.

D’Alba L, Kieffer L, Shawkey MD (2012) Relative contributions of pigments and biophotonic nanostructures to natural color production: A case study in budgerigar (Melopsittacus undulatus) feathers. Journal of Experimental Biology, 215, 1272-1277.

Davies BH (1985) Carotenoid metabolism in animals: A biochemist's view. Pure and Applied Chemistry, 57,
679-684.

del Val E, Senar JC, Garrido-Fernández J, Jarén M, Borràs A, Cabrera J, Negro JJ (2009) The liver but not the skin is the site for conversion of a red carotenoid in a passerine bird. Naturwissenschaften, 96, 797-801.

During A, Dawson HD, Harrison EH (2005) Carotenoid transport is decreased and expression of the lipid transporters SR-BI, NPC1L1, and ABCA1 is downregulated in Caco-2 cells treated with ezetimibe. Journal of Nutrition, 135, 2305-2312.

Erdman JW, Bierer TL, Gugger ET (1993) Absorption and transport of carotenoids. Annals of the New York Academy of Sciences, 691, 76-85.

Eriksson J, Larson G, Gunnarsson U, Bed'Hom B, Tixier-Boichard M, Strömstedt L, Wright D, Jungerius A, Vereijken A, Randi E, Jensen P, Andersson L (2008) Identification of the yellow skin gene reveals a hybrid origin of the domestic chicken. PLoS Genetics, 4, e10000100.

Gao GQ, Xu M, Bai CL, Yang YL, Li GP, Xu JY, Wei ZY, Min JM, Su GH, Zhou XQ, Guo J, Hao Y, Zhang GP, Yang XK, Xu XM, Widelitz RB, Chuong CM, Zhang C, Yin J, Zuo YC (2018) Comparative genomics and transcriptomics of Chrysolophus provide insights into the evolution of complex plumage colouration. GigaScience, 7, 1-14.

Gao GQ, Zuo YC, Bai CL, Wei ZY, Hu TM, Li GP (2016) The synthesis and regulatory mechanisms of avian plumage pigmentation. Chinese Journal of Zoology, 51, 323-335. (in Chinese with English abstract) [高广琦, 左永春, 白春玲, 魏著英, 扈廷茂, 李光鹏 (2016) 鸟类羽毛色素的合成机 理与调控机制. 动物学杂志, 51, 323-335.]

Gazda MA, Araújo PM, Lopes RJ, Toomey MB, Andrade P, Afonso S, Marques C, Nunes L, Pereira P, Trigo S, Hill GE, Corbo JC, Carneiro M (2020a) A genetic mechanism for sexual dichromatism in birds. Science, 368, 1270-1274.

Gazda MA, Toomey MB, Araújo PM, Lopes RJ, Afonso S, Myers CA, Serres K, Kiser PD, Hill GE, Corbo JC, Carneiro M (2020b) Genetic basis of de novo appearance of carotenoid ornamentation in bare parts of canaries. Molecular Biology and Evolution, 37, 1317-1328.

Ge ZY, Johnson JD, Cobine PA, McGraw KJ, Garcia R, Hill GE (2015) High concentrations of ketocarotenoids in hepatic mitochondria of Haemorhous mexicanus. Physiological and Biochemical Zoology, 88, 444-450.

Hamilton WD, Zuk M (1982) Heritable true fitness and bright birds: A role for parasites? Science, 218, 384-387.

Hartley RC, Kennedy MW (2004) Are carotenoids a red herring in sexual display? Trends in Ecology \& Evolution, 19, 353-354.

Herron KL, McGrane MM, Waters D, Lofgren IE, Clark RM, Ordovas JM, Fernandez ML (2006) The ABCG5 polymorphism contributes to individual responses to dietary cholesterol and carotenoids in eggs. Journal of Nutrition, 136, 1161-1165. 
Hill GE (2011) Condition-dependent traits as signals of the functionality of vital cellular processes. Ecology Letters, 14, 625-634.

Hill GE, Hood WR, Ge ZY, Grinter R, Greening C, Johnson JD, Park NR, Taylor HA, Andreasen VA, Powers MJ, Justyn NM, Parry HA, Kavazis AN, Zhang YF (2019) Plumage redness signals mitochondrial function in the house finch. Proceedings of the Royal Society B: Biological Sciences, 286, 20191354.

Hill GE, Johnson JD (2012) The vitamin A-redox hypothesis: A biochemical basis for honest signaling via carotenoid pigmentation. The American Naturalist, 180, E127-E150.

Hill GE, McGraw KJ (2006) Bird Coloration, Volume I: Mechanisms and Measurements. Harvard University Press, Cambridge, USA.

Hill GE, Montgomerie R, Inouye CY, Dale J (1994) Influence of dietary carotenoids on plasma and plumage colour in the house finch: Intra- and intersexual variation. Functional Ecology, 8, 343-350.

Hubbard JK, Uy JAC, Hauber ME, Hoekstra HE, Safran RJ (2010) Vertebrate pigmentation: From underlying genes to adaptive function. Trends in Genetics, 26, 231-239.

Hudon J (1991) Unusual carotenoid use by the Western Tanager (Piranga ludoviciana) and its evolutionary implications. Canadian Journal of Zoology, 69, 2311-2320.

Huggins KA, Navara KJ, Mendonça MT, Hill GE (2010) Detrimental effects of carotenoid pigments: The dark side of bright coloration. Naturwissenschaften, 97, 637-644.

Hui BD (2005) Carotenoid chemistry and biochemistry. China Light Industry Press, Beijing. (in Chinese) [惠伯棣 (2005) 类胡萝卜素化学及生物化学. 中国轻工业出版社, 北京.]

Inouye CY, Hill GE, Stradi RD, Montgomerie R (2001) Carotenoid pigments in male house finch plumage in relation to age, subspecies, and ornamental coloration. The Auk, 118, 900-915.

Johnson JD, Hill GE (2013) Is carotenoid ornamentation linked to the inner mitochondria membrane potential? A hypothesis for the maintenance of signal honesty. Biochimie, 95, 436-444.

Kerje S, Lind J, Schütz K, Jensen P, Andersson L (2003) Melanocortin 1-receptor (MC1R) mutations are associated with plumage colour in chicken. Animal Genetics, 34, 241-248.

Khalil S, Welklin JF, McGraw KJ, Boersma J, Schwabl H, Webster MS, Karubian J (2020) Testosterone regulates CYP2J19-linked carotenoid signal expression in male red-backed fairywrens (Malurus melanocephalus). Proceedings of the Royal Society B: Biological Sciences, 287, 20201687.

Kiefer C, Sumser E, Wernet M (2002) A class B scavenger receptor mediates the cellular uptake of carotenoids in Drosophila. Proceedings of the National Academy of Sciences, USA, 99, 10581-10586.
Koch RE, Kavazis AN, Hasselquist D, Hood WR, Zhang YF, Toomey MB, Hill GE (2018) No evidence that carotenoid pigments boost either immune or antioxidant defenses in a songbird. Nature Communications, 9, 491.

Koch RE, McGraw KJ, Hill GE (2016) Effects of diet on plumage coloration and carotenoid deposition in red and yellow domestic canaries (Serinus canaria). The Wilson Journal of Ornithology, 128, 328-333.

Kodric-Brown A, Brown JH (1984) Truth in advertising: The kinds of traits favored by sexual selection. The American Naturalist, 124, 309-323.

Kotake-Nara E, Nagao A (2011) Absorption and metabolism of xanthophylls. Marine Drugs, 9, 1024-1037.

LaFountain AM, Frank HA, Prum RO (2013) Carotenoids from the crimson and maroon plumages of Old World orioles (Oriolidae). Archives of Biochemistry and Biophysics, 539, 126-132.

LaFountain AM, Kaligotla S, Cawley S, Riedl KM, Schwartz SJ, Frank HA, Prum RO (2010) Novel methoxy-carotenoids from the burgundy-colored plumage of the Pompadour Cotinga Xipholena punicea. Archives of Biochemistry and Biophysics, 504, 142-153.

LaFountain AM, Prum RO, Frank HA (2015) Diversity, physiology, and evolution of avian plumage carotenoids and the role of carotenoid-protein interactions in plumage color appearance. Archives of Biochemistry and Biophysics, 572, 201-212.

Ligon RA, Simpson RK, Mason NA, Hill GE, McGraw KJ (2016) Evolutionary innovation and diversification of carotenoid-based pigmentation in finches. Evolution, 70, 2839-2852.

Lopes RJ, Johnson JD, Toomey MB, Ferreira MS, Araujo PM, Melo-Ferreira J, Andersson L, Hill GE, Corbo JC, Carneiro M (2016) Genetic basis for red coloration in birds. Current Biology, 26, 1427-1434.

Maia R, Macedo RHF, Shawkey MD (2012) Nanostructural self-assembly of iridescent feather barbules through depletion attraction of melanosomes during keratinization. Journal of the Royal Society Interface, 9, 734-743.

McGraw KJ, Hill GE, Parker RS (2003) Carotenoid pigments in a mutant cardinal: Implications for the genetic and enzymatic control mechanisms of carotenoid metabolism in birds. The Condor, 105, 587-592.

McGraw KJ, Hill GE, Parker RS (2005) The physiological costs of being colourful: Nutritional control of carotenoid utilization in the American goldfinch, Carduelis tristis. Animal Behaviour, 69, 653-660.

McGraw KJ, Nolan PM, Crino OL (2006) Carotenoid accumulation strategies for becoming a colourful house finch: Analyses of plasma and liver pigments in wild moulting birds. Functional Ecology, 20, 678-688.

Mendes-Pinto MM, LaFountain AM, Stoddard MC, Prum RO, Frank HA, Robert B (2012) Variation in carotenoid-protein 
interaction in bird feathers produces novel plumage coloration. Journal of the Royal Society Interface, 9, 3338-3350.

Morehouse NI (2014) Condition-dependent ornaments, life histories, and the evolving architecture of resource-use. Integrative and Comparative Biology, 54, 591-600.

Mundy NI (2018) Colouration genetics: Pretty polymorphic parrots. Current Biology, 28, R113-R114.

Mundy NI, Stapley J, Bennison C, Tucker R, Twyman H, Kim KW, Burke T, Birkhead TR, Andersson S, Slate J (2016) Red carotenoid coloration in the zebra finch is controlled by a cytochrome P450 gene cluster. Current Biology, 26, 1435-1440.

Nadeau NJ, Minvielle FF, Ito SI, Inoue-Murayama $M$, Gourichon DD, Follett SA, Burke T, Mundy NI (2008) Characterization of Japanese quail yellow as a genomic deletion upstream of the avian homolog of the mammalian ASIP (agouti) gene. Genetics, 178, 777-786.

Nagao A (2011) Absorption and metabolism of dietary carotenoids. BioFactors, 37, 83-87.

Olson VA, Owens IPF (1998) Costly sexual signals: Are carotenoids rare, risky or required? Trends in Ecology \& Evolution, 13, 510-514.

Orteu A, Jiggins CD (2020) The genomics of coloration provides insights into adaptive evolution. Nature Reviews Genetics, 21, 461-475.

Parker RS (1996) Absorption, metabolism, and transport of carotenoids. The FASEB Journal, 10, 542-551.

Prum RO, LaFountain AM, Berg CJ, Tauber MJ, Frank HA (2014) Mechanism of carotenoid coloration in the brightly colored plumages of broadbills (Eurylaimidae). Journal of Comparative Physiology B: Biochemical Systemic and Environmental Physiology, 184, 651-672.

Prum RO, LaFountain AM, Berro J, Stoddard MC, Frank HA (2012) Molecular diversity, metabolic transformation, and evolution of carotenoid feather pigments in cotingas (Aves: Cotingidae). Journal of Comparative Physiology B: Biochemical Systemic and Environmental Physiology, 182, 1095-1116.

Sakudoh T, Kuwazaki S, Iizuka T, Narukawa J, Yamamoto K, Uchino K, Sezutsu H, Banno Y, Tsuchida K (2013) CD36 homolog divergence is responsible for the selectivity of carotenoid species migration to the silk gland of the silkworm Bombyx mori. Journal of Lipid Research, 54, 482-495.

Scita G, Aponte GW, Wolf G (1993) Uptake and cleavage of beta-carotene by cultures of rat small intestinal cells and human lung fibroblasts. Methods in Enzymology, 214, 21-32.

Shawkey MD, D’Alba L (2017) Interactions between colour-producing mechanisms and their effects on the integumentary colour palette. Philosophical Transactions of the Royal Society B: Biological Sciences, 372, 20160536.
Shawkey MD, Hill GE (2005) Carotenoids need structural colours to shine. Biology Letters, 1, 121-124.

Stradi R, Rossi E, Celentano G, Bellardi B (1996) Carotenoids in bird plumage: The pattern in three Loxia species and in Pinicola enucleator. Comparative Biochemistry and Physiology B: Biochemistry \& Molecular Biology, 113, 427-432.

Svensson PA, Wong B (2011) Carotenoid-based signals in behavioural ecology: A review. Behaviour, 148, 131-189.

Thomas DB, McGraw KJ, Butler MW, Carrano MT, Madden O, James HF (2014) Ancient origins and multiple appearances of carotenoid-pigmented feathers in birds. Proceedings of the Royal Society B: Biological Sciences, 281, 20140806.

Tobita-Teramoto T, Jang GY, Kino K, Salter DW, Brumbaugh J, Akiyama T (2000) Autosomal albino chicken mutation (c(a)/c(a)) deletes hexanucleotide (- $\Delta$ GACTGG817) at a copper-binding site of the tyrosinase gene. Poultry Science, 79, 46-50.

Toews DPL, Hofmeister NR, Taylor SA (2017) The evolution and genetics of carotenoid processing in animals. Trends in Genetics, 33, 171-182.

Toews DPL, Taylor SA, Vallender R, Brelsford A, Butcher BG, Messer PW, Lovette IJ (2016) Plumage genes and little else distinguish the genomes of hybridizing warblers. Current Biology, 26, 2313-2318.

Toomey MB, Lopes RJ, Araujo PM, Johnson JD, Gazda MA, Afonso S, Mota PG, Koch RE, Hill GE, Corbo JC, Carneiro M (2017) High-density lipoprotein receptor SCARB1 is required for carotenoid coloration in birds. Proceedings of the National Academy of Sciences, USA, 114, 5219-5224.

Twyman H, Andersson S, Mundy NI (2018a) Evolution of CYP2J19, a gene involved in colour vision and red coloration in birds: Positive selection in the face of conservation and pleiotropy. BMC Evolutionary Biology, 18, 22.

Twyman H, Prager M, Mundy NI, Andersson S (2018b) Expression of a carotenoid-modifying gene and evolution of red coloration in weaverbirds (Ploceidae). Molecular Ecology, 27, 449-458.

Twyman H, Valenzuela N, Literman R, Andersson S, Mundy NI (2016) Seeing red to being red: Conserved genetic mechanism for red cone oil droplets and co-option for red coloration in birds and turtles. Proceedings of the Royal Society B: Biological Sciences, 283, 20161208.

Va ge DI, Boman IA (2010) A nonsense mutation in the beta-carotene oxygenase 2 ( $\mathrm{BCO} 2)$ gene is tightly associated with accumulation of carotenoids in adipose tissue in sheep (Ovis aries). BMC Genetics, 11, 10.

Van der Horst DJ, Roosendaal SD, Rodenburg KW (2009) Circulatory lipid transport: Lipoprotein assembly and function from an evolutionary perspective. Molecular and Cellular Biochemistry, 326, 105-119. 
von Lintig J (2010) Colors with functions: Elucidating the biochemical and molecular basis of carotenoid metabolism. Annual Review of Nutrition, 30, 35-56.

von Lintig J, Moon J, Lee J, Ramkumar S (2020) Carotenoid metabolism at the intestinal barrier. BBA-Molecular and Cell Biology of Lipids, 1865, 158580.

von Schantz T, Bensch S, Grahn M, Hasselquist D, Wittzell H (1999) Good genes, oxidative stress and condition-dependent sexual signals. Proceedings of the Royal Society B: Biological Sciences, 266, 1-12.

Walsh N, Dale J, McGraw KJ, Pointer MA, Mundy NI (2012) Candidate genes for carotenoid coloration in vertebrates and their expression profiles in the carotenoid-containing plumage and bill of a wild bird. Proceedings of the Royal Society B: Biological Sciences, 279, 58-66.

Weaver RJ, Koch RE, Hill GE (2017) What maintains signal honesty in animal colour displays used in mate choice? Philosophical Transactions of the Royal Society B: Biological Sciences, 372, 20160343.

Weaver RJ, Santos ESA, Tucker AM, Wilson AE, Hill GE (2018) Carotenoid metabolism strengthens the link between feather coloration and individual quality. Nature Communications, 9, 73.

Weesie RJ, Askin D, Jansen FJHM, de Groot HJM, Lugtenburg J, Britton G (1995) Protein-chromophore interactions in $\alpha$-crustacyanin, the major blue carotenoprotein from the carapace of the lobster, Homarus gammarus. A study by ${ }^{13} \mathrm{C}$ magic angle spinning NMR. FEBS Letters, 362, 34-38.

Yeum KJ, Russell RM (2002) Carotenoid bioavailability and bioconversion. Annual Review of Nutrition, 22, 483-504.

Yu LQ, von Bergmann K, Lutjohann D, Hobbs HH, Cohen JC (2004) Selective sterol accumulation in ABCG5/ABCG8deficient mice. Journal of Lipid Research, 45, 301-307.

Zahavi A (1977) The cost of honesty: Further remarks on the handicap principle. Journal of Theoretical Biology, 67, 603-605.

Zheng GM (2012) Ornithology, 2nd edn. Beijing Normal University Press, Beijing. (in Chinese) [郑光美 (2012) 鸟 类学(第二版). 北京师范大学出版社, 北京.]

(责任编委: 李晟 责任编辑: 间文杰) 